\title{
Prevalence, incidence and characteristics of chronic cough among adults from the Canadian Longitudinal Study on Aging
}

\author{
Imran Satia (10) 1,2,3,4, Alexandra J. Mayhew ${ }^{3,4}$, Nazmul Sohel ${ }^{3,4}$, \\ Om Kurmi (1) 1,2,3,5, Kieran J. Killian ${ }^{1}$, Paul M. O’Byrne (ib) ${ }^{1,2}$ and Parminder Raina ${ }^{3,4}$
}

Affiliations: ${ }^{1}$ Dept of Medicine, McMaster University, Hamilton, ON, Canada. ${ }^{2}$ Firestone Institute for Respiratory Health, St Joseph's Healthcare, Hamilton, ON, Canada. ${ }^{3}$ Dept of Health Research Methods, Evidence, and Impact, Faculty of Health Sciences, McMaster University, Hamilton, ON, Canada. ${ }^{4}$ McMaster Institute for Research on Aging, McMaster University, Hamilton, ON, Canada. ${ }^{5}$ Faculty of Health and Life Sciences, Coventry University, Coventry, UK.

Correspondence: Imran Satia, Dept of Medicine, Division of Respirology, McMaster University, 1280 Main St West, Hamilton, Ontario, L8S 4L8, Canada. E-mail: satiaiđmcmaster.ca

ABSTRACT The global prevalence of chronic cough is highly variable, ranging from $2 \%$ to $18 \%$. There is a lack of data on the prevalence and incidence of chronic cough in the general population. The objective of this study was to investigate the prevalence and incidence of chronic cough in a sample of Canadian adults, and how these are influenced by age, sex, smoking, respiratory symptoms, medical comorbidities and lung function.

Participants with chronic cough were identified from the Canadian Longitudinal Study on Aging (CLSA) based on self-reported daily cough in the past 12 months. This is a prospective, nationally generalisable, stratified random sample of adults aged 45-85 years at baseline recruited between 2011 and 2015, and followed-up 3 years later. The prevalence and incidence per 100 person-years are described, with adjustments for age, sex and smoking.

Of the 30097 participants, 29972 completed the chronic cough question at baseline and 26701 did so at follow-up. The prevalence of chronic cough was $15.8 \%$ at baseline and $17.6 \%$ at follow-up with $10.4-17.1 \%$ variation across seven provinces included in the CLSA comprehensive sample. Prevalence increased with age and current smoking, and was higher in males (15.2\%), Caucasians (14\%) and those born in North America, Europe or Oceania (14\%). The incidence of chronic cough adjusted for age, sex and smoking was higher in males and in underweight and obese subjects. Subjects with respiratory symptoms, airway diseases, lower forced expiratory volume in $1 \mathrm{~s}$ (\% predicted), cardiovascular diseases, psychological disorders, diabetes and chronic pain had a higher incidence of chronic cough.

The prevalence and incidence of chronic cough is high in the CLSA sample with geographic, ethnic and gender differences, influenced by a number of medical comorbidities.

@ERSpublications

Canada has one of the highest prevalences of chronic cough in world and it is more common in Caucasians of European descent. Ageing, smoking, sex, clinical comorbidities and lung physiology all influence the prevalence and incidence of chronic cough. https://bit.ly/3qSBkdp

Cite this article as: Satia I, Mayhew AJ, Sohel N, et al. Prevalence, incidence and characteristics of chronic cough among adults from the Canadian Longitudinal Study on Aging. ERJ Open Res 2021; 7: 00160-2021 [https://doi.org/10.1183/23120541.00160-2021]. 


\section{Introduction}

Chronic cough, defined as a daily cough lasting $>8$ weeks, is a prevalent condition associated with poor quality of life [1-4]. The estimated $10 \%$ of adults globally with chronic cough report adverse psychosocial and physical effects such as exhaustion, depression and disruptions to social interactions, sleep and work [5-8]. Chronic cough is one of the most common reasons for referral to a specialist in secondary care, representing a significant burden on the healthcare system [9, 10]. Quantitative assessments of the prevalence, incidence and characteristic features of chronic cough help to identify at-risk populations and potentially causative factors, and reflects the burden of chronic cough in the general population.

The global prevalence of chronic cough is highly variable by geographic region, ranging between $2 \%$ and $18 \%$ in a systematic review and meta-analysis of 90 studies [5]. The differences by region persisted after adjustment for smoking and highlight the importance of country-specific estimates of the prevalence of chronic cough. The majority of the studies did not include chronic cough as a primary outcome and therefore did not examine the prevalence of cough stratified by demographic, physiological and clinical variables. These can influence the prevalence and incidence of chronic cough. Furthermore, there is a lack of data describing the incidence of chronic cough from large nationwide study.

The objective of this study was to estimate the prevalence and incidence of chronic cough in a national sample of adults from the Canadian Longitudinal Study on Aging (CLSA) who were between the ages of 45 and 85 years at the baseline and to assess how prevalence and incidence differs by age, sex, smoking, respiratory symptoms, comorbidities and lung function.

\section{Study design and methods}

\section{Study design and population}

The CLSA is a large, nationally generalisable, stratified random sample of 51338 Canadian men and women aged 45-85 years at baseline (2011-2015) from the 10 Canadian provinces [11]. Eligible participants had to be physically and cognitively able to participate on their own and not living in institutions such as long-term care facilities. Participants were recruited in the tracking cohort $(n=21241)$ and the comprehensive cohort $(n=30097)$. Tracking-cohort participants were randomly selected from the 10 provinces and completed interviews by phone. Participants in the comprehensive cohort were randomly selected from within $25-50 \mathrm{~km}$ of 11 data collection sites, located in seven provinces $(\mathrm{n}=30097)$. In addition to completing interviews in person, the comprehensive participants completed in-depth physical assessments and provided blood and urine samples. Details of the study design have been described elsewhere [12]. Each participant is followed every 3 years for 20 years or until death. The first follow-up was conducted between 2015 and 2018 with a retention rate of 95\%. The comprehensive data from baseline and follow-up 1 were included in the current analyses. This study was approved by the Hamilton integrated research ethics board and by the CLSA scientific advisory board (project ID: 1909024).

\section{Chronic cough definitions}

Participants who self-identified as having coughed most days within the past 12 months were categorised as having a chronic cough at baseline and at follow-up. Chronic cough was further classified as a productive cough if participants reported bringing up phlegm in the morning or most days during the year. Participants who did not report the presence of phlegm were categorised as having a dry cough. Questionnaires were available in both English and French.

\section{Respiratory symptoms and chronic conditions}

Self-reported presence of respiratory symptoms including chest pain, shortness of breath upon exertion and wheezing were assessed. Participants were asked "Do you wheeze with mild to moderate exertion?", "Do you become short of breath climbing stairs or walking up a small hill?" and "Do you get chest pain or discomfort when you walk uphill or hurry?".

Disease definitions were based on self-reported physician diagnosis on direct questioning of participants by trained research assistants at baseline and at each follow-up. Participants were asked if they had ever been diagnosed with a list of respiratory, cardiovascular, metabolic, neurological, musculoskeletal and mental health disorders. A history of infectious disease such as pneumonia or influenza in the past 12 months was also surveyed.

\section{Spirometry}

Lung function was measured with the TruFlow Easy-On Air Spirometer (ndd Medical Technologies, Zurich, Switzerland) and categorised based on the American Thoracic Society requirements and criteria. Participants screened positive for major contraindications were excluded [13]. The highest forced expiratory volume in $1 \mathrm{~s}\left(\mathrm{FEV}_{1}\right)$ and forced vital capacity $(\mathrm{FVC})$ from three acceptable maximal efforts 
were selected. Only grades A and B were accepted for analysis. The $\mathrm{FEV}_{1}, \mathrm{FVC}$ and $\mathrm{FEV}_{1} / \mathrm{FVC}$ ratio was recorded without bronchodilator therapy.

Chronic airflow obstruction was defined as a $\mathrm{FEV}_{1} / \mathrm{FVC}$ ratio of $<0.7$ as well as using the lower limit of normal (LLN). Age, height and sex were used to develop CLSA-specific prediction reference values for this total population. These were based on standard allometric principles, $\mathrm{FEV}_{1}$ and FVC increase in a positively accelerating manner with height $(\mathrm{y}=\mathrm{k} \times$ HeightK1) there was a proportionate increase in males relative to females at the same height $\left(\mathrm{y}=\mathrm{k} \times \operatorname{Height}_{1} \times\left(1+\mathrm{K}_{2} \times\right.\right.$ Males(1))); and decreasing by a constant proportion with age $\left(\mathrm{y}=\mathrm{k} \times \operatorname{Height}_{1} \times\left(1+\mathrm{K}_{2} \times \operatorname{Males}(1) \times\left(1-\mathrm{K}_{3} \times(\right.\right.\right.$ Age $\left.\left.\left.)\right)\right)\right)$. Grade $\mathrm{A}$ and $\mathrm{B}$ spirometry data were available in 22547 participants.

\section{Statistical analysis}

Descriptive statistics for demographic and health variables stratified by chronic cough status were reported as mean $\pm \mathrm{SE}$ for continuous variables and frequencies and percentages for categorical variables. The prevalence of chronic cough was assessed by age group, sex, smoking status, respiratory symptoms, $\mathrm{FEV}_{1} \%$ predicted categories and chronic airway obstruction, and by the presence of asthma, COPD or both asthma and COPD. The incidence of chronic cough per 100 person-years was calculated (incidence = (new cases/ total follow-up years $) \times 100)$ in the 22547 participants who did not report the presence of chronic cough at baseline and provided chronic cough information at follow-up time. Incidence rates were stratified by the same variables used to assess the prevalence of chronic cough as well as body mass index categories, the presence of common cardiorespiratory, vascular, gastrointestinal, metabolic, neurological and mental health disorders.

The CLSA provided inflation weights, which were used for all analyses (CLSA sample weight version 1.2). The inflation weights are proportional to the reciprocal of the individual inclusion probabilities and are recalibrated to the Canadian population within strata of sex, age and geographic area [14]. Using the inflation weights allows the results to be generalisable to the catchment areas of the 11 data collection sites located in seven provinces (CLSA Methodology Working Group 2017). The survey analysis procedure in SAS was used to account for the stratified sampling technique. All analyses were conducted using SAS version 9.4 .

\section{Results}

\section{Study population and demographics}

The comprehensive cohort included 30097 participants. A total of 29972 completed the chronic cough question at baseline (table 1) and $26701 \mathrm{did}$ so at follow-up. Of these, 4739 (15.8\%) participants reported chronic cough at baseline and $4694(17.6 \%)$ at follow-up. The mean age of participants reporting cough was 61.5 years with more males than females reporting chronic cough at baseline ( $15.2 \%$ versus $12.7 \%)$ and at follow-up (16.2\% and $14.4 \%)$. The majority of participants with and without cough were overweight at baseline. The prevalence of chronic cough was greatest in the underweight (19.6\%) and obese (17.0\%) categories. There were 2558 (8.5\%) current smokers at baseline and participants with chronic cough had a two-fold higher smoking pack-year history (mean 6.7 versus 13.2 pack-years) with a prevalence of chronic cough of $34 \%$ in current smokers.

\section{Differences across Canadian provinces, ethnic origin and country of birth}

There were provincial differences in the prevalence of chronic cough across Canada (table 1). Participants from Manitoba (17.1\%) had the highest prevalence of chronic cough, and Quebec the lowest prevalence at baseline (10.4\%). $>90 \%$ of the CLSA participants were white, with smaller representation from other ethnic backgrounds. Participants from a white background had the highest prevalence (14.0\%), while black, South Asian, Asian and Arab backgrounds ranged from $5 \%$ to $8 \%$. $~ 84 \%$ were born in Canada and the USA, and along with those born in Europe and Oceania, all demonstrated a similar prevalence of $\sim 14 \%$.

\section{Effects of ageing, sex and smoking on the prevalence of chronic cough at baseline}

The prevalence of chronic cough was greatest in current smokers, ranging from $27 \%$ to $44 \%$, and was lower in former $(7-21 \%)$ or nonsmokers $(6-15 \%)$ (figure 1). The prevalence of chronic cough increased with age, was higher in males and was highest in current smokers in both sexes across all age groups.

\section{Chronic cough-associated respiratory airways diseases, symptoms and physiology}

In participants who complained of chronic cough, dry cough was reported by $51 \%$, while $43 \%$ had cough with phlegm (6\% did not report if phlegm was present or not). Participants reporting respiratory symptoms at baseline, lower $\mathrm{FEV}_{1} \%$ pred, airflow obstruction (both $\mathrm{FEV}_{1} / \mathrm{FVC}$ ratio $<0.7$ or $<\mathrm{LLN}$ ) or the presence of COPD, asthma or both, had a higher prevalence of chronic cough at baseline (figure 2). 


\section{TABLE 1 Demographics of chronic cough at baseline ${ }^{\#}$}

\begin{tabular}{|c|c|c|c|c|}
\hline \multirow[b]{2}{*}{ Subjects } & \multicolumn{2}{|c|}{ No chronic cough } & \multicolumn{2}{|c|}{ Chronic cough } \\
\hline & 25233 & & 4739 & \\
\hline Age years & & $59.2 \pm 0.04$ & & $61.5 \pm 0.18$ \\
\hline \multicolumn{5}{|l|}{ Sex } \\
\hline Male & 12184 & $84.8 \pm 0.37$ & 2526 & $15.2 \pm 0.37$ \\
\hline Female & 13049 & $87.3 \pm 0.31$ & 2213 & $12.7 \pm 0.31$ \\
\hline BMI $\mathrm{kg} \cdot \mathrm{m}^{-2}$ & 25139 & $27.7 \pm 0.04$ & 4701 & $28.6 \pm 0.11$ \\
\hline Underweight <20 & 171 & $80.4 \pm 3.24$ & 42 & $19.6 \pm 3.24$ \\
\hline Normal $20-25$ & 7644 & $88.0 \pm 0.41$ & 1190 & $12.0 \pm 0.41$ \\
\hline Overweight 25-30 & 10221 & $86.7 \pm 0.37$ & 1815 & $13.3 \pm 0.37$ \\
\hline Obese $>30$ & 7103 & $83.0 \pm 0.49$ & 1654 & $17.0 \pm 0.49$ \\
\hline \multicolumn{5}{|l|}{ Smoking status } \\
\hline Never & 13729 & $89.1 \pm 0.30$ & 1953 & $10.9 \pm 0.30$ \\
\hline Former & 9755 & $86.5 \pm 0.38$ & 1803 & $13.5 \pm 0.38$ \\
\hline Current & 1605 & $65.8 \pm 1.17$ & 953 & $34.2 \pm 1.17$ \\
\hline Smoking packs per year & 24952 & $6.7 \pm 0.10$ & 4680 & $13.2 \pm 0.35$ \\
\hline \multicolumn{5}{|l|}{ Provinces } \\
\hline Alberta & 2445 & $85.7 \pm 0.76$ & 493 & $14.3 \pm 0.76$ \\
\hline British Columbia & 5147 & $84.2 \pm 0.51$ & 1075 & $15.8 \pm 0.51$ \\
\hline Manitoba & 2548 & $82.9 \pm 0.75$ & 552 & $17.1 \pm 0.75$ \\
\hline Newfoundland and Labrador & 1880 & $86.0 \pm 0.80$ & 331 & $14.0 \pm 0.80$ \\
\hline Nova Scotia & 2547 & $84.7 \pm 0.70$ & 511 & $15.3 \pm 0.70$ \\
\hline Ontario & 5320 & $84.2 \pm 0.49$ & 1087 & $15.8 \pm 0.49$ \\
\hline Quebec & 5346 & $89.6 \pm 0.42$ & 690 & $10.4 \pm 0.42$ \\
\hline \multicolumn{5}{|l|}{ Ethnicity } \\
\hline White only & 22948 & $86.0 \pm 0.25$ & 4359 & $14.0 \pm 0.25$ \\
\hline Black only & 186 & $94.5 \pm 1.58$ & 17 & $5.5 \pm 1.58$ \\
\hline Asian (East and West) & 355 & $91.7 \pm 1.87$ & 31 & $8.3 \pm 1.87$ \\
\hline South Asian & 245 & $92.0 \pm 1.84$ & 25 & $8.0 \pm 1.84$ \\
\hline Arab & 79 & $93.0 \pm 3.15$ & 8 & $7.0 \pm 3.15$ \\
\hline Others & 1213 & $83.2 \pm 1.22$ & 254 & $16.8 \pm 1.22$ \\
\hline \multicolumn{5}{|l|}{ Country of birth } \\
\hline Canada & 20579 & $85.5 \pm 0.27$ & 3980 & $14.5 \pm 0.27$ \\
\hline USA & 568 & $86.4 \pm 1.48$ & 116 & $13.6 \pm 1.48$ \\
\hline South, Central America and Caribbean & 378 & $92.0 \pm 1.49$ & 41 & $8.0 \pm 1.49$ \\
\hline Europe/Oceania & 2846 & $86.6 \pm 0.69$ & 522 & $13.4 \pm 0.69$ \\
\hline Africa & 257 & $92.3 \pm 1.71$ & 27 & $7.7 \pm 1.71$ \\
\hline Asia & 601 & $93.2 \pm 1.22$ & 52 & $6.8 \pm 1.22$ \\
\hline
\end{tabular}

Data are presented as $n$, mean \pm SE or $\% \pm$ SE. BMI: body mass index. ${ }^{\#}: \mathrm{n}=29972$.

A $\mathrm{FEV}_{1}$ of $<50 \%$ pred, combined asthma and COPD or COPD alone and wheeze had the highest prevalence. The prevalence of chronic cough in COPD was almost twice that of asthma.

\section{Factors affecting the incidence of chronic cough}

26701 participants attended for follow-up 1 (3271 lost to follow-up), and 2506 participants developed chronic cough between baseline and follow-up. The yearly incidence rate per 100 person-years of chronic cough increased from the youngest age group (45-54 years; $2.60,95 \%$ CI $2.33-2.86$ ) to the oldest age group (75-85 years; $4.50,4.05-4.95)$ was higher in males than in females, in current smokers compared with former smokers or never-smokers, and highest in underweight and obese participants (table 2). After adjustment for age, sex and smoking, the same patterns were observed.

Adjusted incidence rates per 100 person-years of chronic cough were highest in the presence of wheezing (7.0), combined asthma/COPD (9.2), COPD (8.0) or asthma alone (5.7), airflow obstruction (either $\mathrm{FEV}_{1} /$ FVC ratio $<0.7$ (5.8) or $<\mathrm{LLN}(6.2)$ ) and lower $\mathrm{FEV}_{1} \%$ pred (figure 3). Adjusted incidence rates were higher in those who had a prior history of stroke (6.8), anxiety (6.2), acute myocardial infarction (6.2), pneumonia (6.4) or influenza (5.8) in the past year, mood disturbance (5.8), current depression (5.7), chronic pain (5.5), migraine (5.5), diabetes (5.5), heart failure (5.6) and history of allergies (5.2) (figure 4). A history of stomach or intestinal ulcers or bowel disorders did not significantly increase the adjusted incidence rates. 


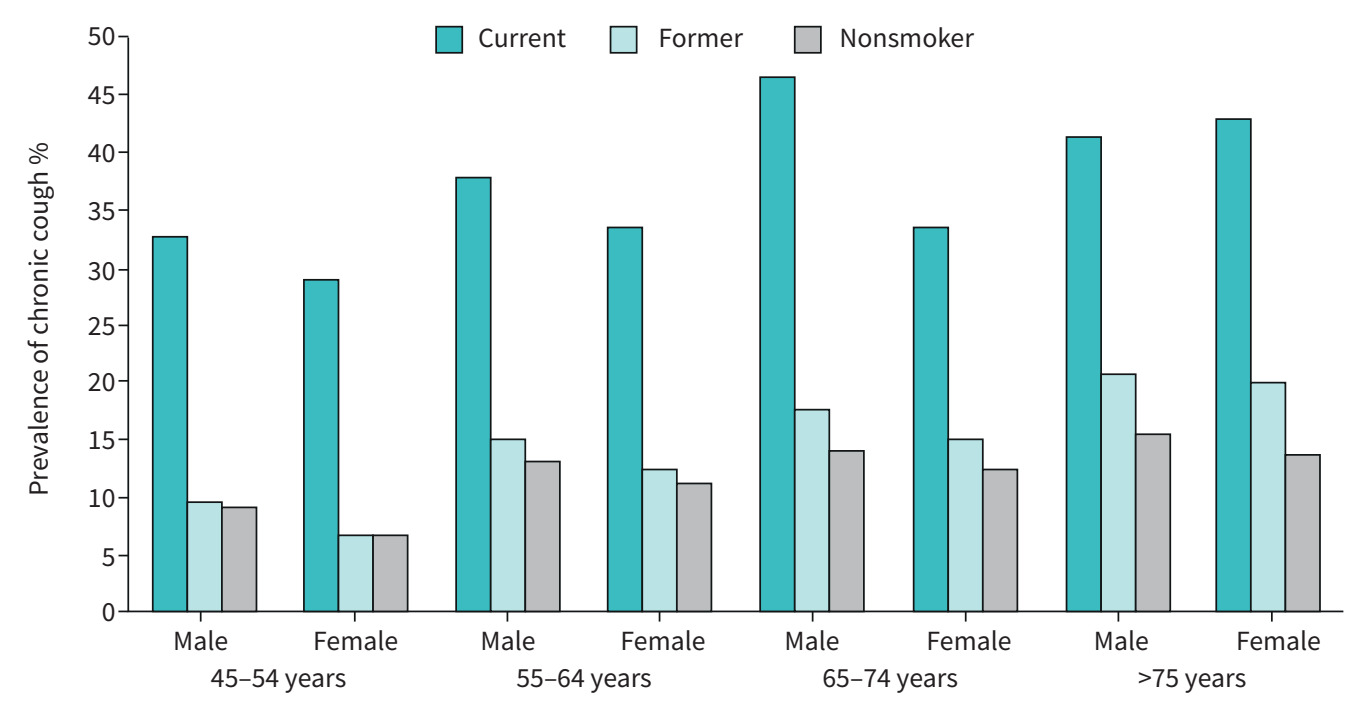

FIGURE 1 Prevalence of chronic cough based on age, sex and smoking status at baseline.

\section{Discussion}

This is the largest population-based study to date describing the prevalence, incidence and characteristics of chronic cough in older adults across a whole country. Approximately 30000 participants were randomly selected and nearly 27000 were followed-up 3 years later. This study demonstrated a high prevalence of chronic cough at baseline and follow-up of $16 \%$ and $18 \%$, respectively, in the older adult population (>45 years), with variations across centres located in seven provinces in Canada. Prevalence increased with age and current smoking and was highest in male, white subjects, and those born in Canada, USA, Europe

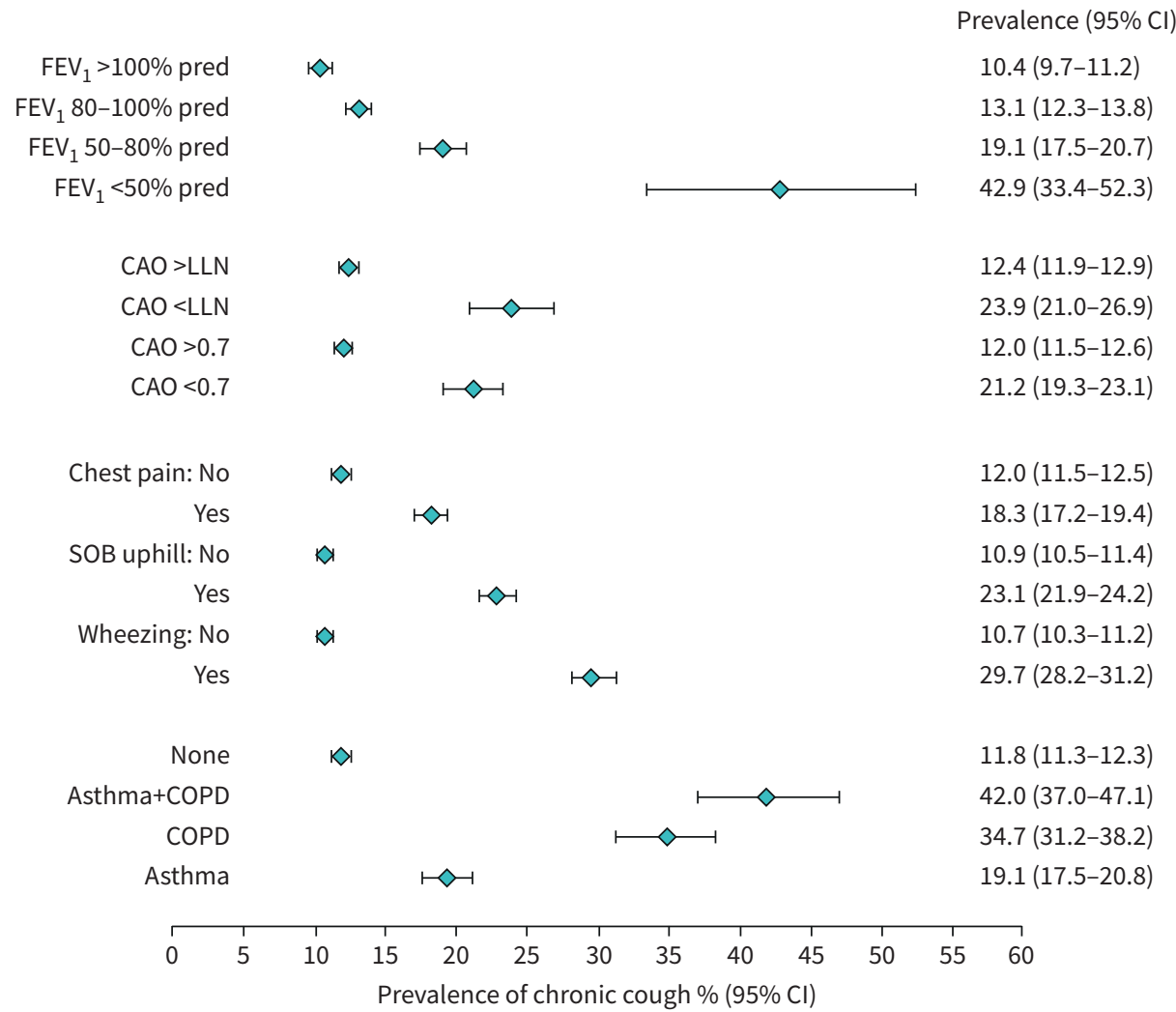

FIGURE 2 The associations of symptoms, lung function, airflow obstruction and airways diseases on the prevalence of chronic cough at baseline. Data are presented as mean (95\% Cl). FEV 1 : forced expiratory volume in $1 \mathrm{~s}$; CAO: chronic airflow obstruction; LLN: lower limit of normal; SOB: shortness of breath. 


\begin{tabular}{|c|c|c|}
\hline & \multicolumn{2}{|c|}{ Incidence rate per 100 person-years } \\
\hline & Unadjusted & Adjusted with age, sex and smoking \\
\hline \multicolumn{3}{|l|}{ Age years } \\
\hline $45-54$ & $2.60(2.33-2.86)$ & $3.58(3.20-3.96)$ \\
\hline $55-64$ & $3.55(3.28-3.82)$ & $4.54(4.17-4.92)$ \\
\hline $65-74$ & $4.20(3.86-4.54)$ & $5.32(4.89-5.75)$ \\
\hline $75-85$ & $4.50(4.05-4.95)$ & $5.70(5.17-6.23)$ \\
\hline \multicolumn{3}{|l|}{ Sex } \\
\hline Male & $3.61(3.37-3.85)$ & $5.09(4.72-5.45)$ \\
\hline Female & $3.06(2.85-3.27)$ & $4.48(4.13-4.83)$ \\
\hline \multicolumn{3}{|l|}{ Smoking } \\
\hline Nonsmoker & $2.86(2.66-3.06)$ & $3.37(3.15-3.58)$ \\
\hline Former & $3.50(3.25-3.76)$ & $3.80(3.53-4.06)$ \\
\hline Current & $6.53(5.65-7.42)$ & $7.20(6.31-8.08)$ \\
\hline \multicolumn{3}{|l|}{ BMI } \\
\hline Underweight & $5.03(1.83-8.23)$ & $5.99(5.97-6.01)$ \\
\hline Normal & $2.53(2.29-2.77)$ & $4.09(4.07-4.11)$ \\
\hline Overweight & $3.41(3.16-3.67)$ & $4.80(4.78-4.82)$ \\
\hline Obese & $4.14(3.81-4.47)$ & $5.60(5.55-5.65)$ \\
\hline
\end{tabular}

and Oceania. The presence of coexisting respiratory symptoms, particularly wheeze, worsening $\mathrm{FEV}_{1} \%$ pred, airflow obstruction, COPD and asthma were all associated with a higher prevalence. The incidence of chronic cough adjusted for age, sex and smoking status was higher with increasing age, current

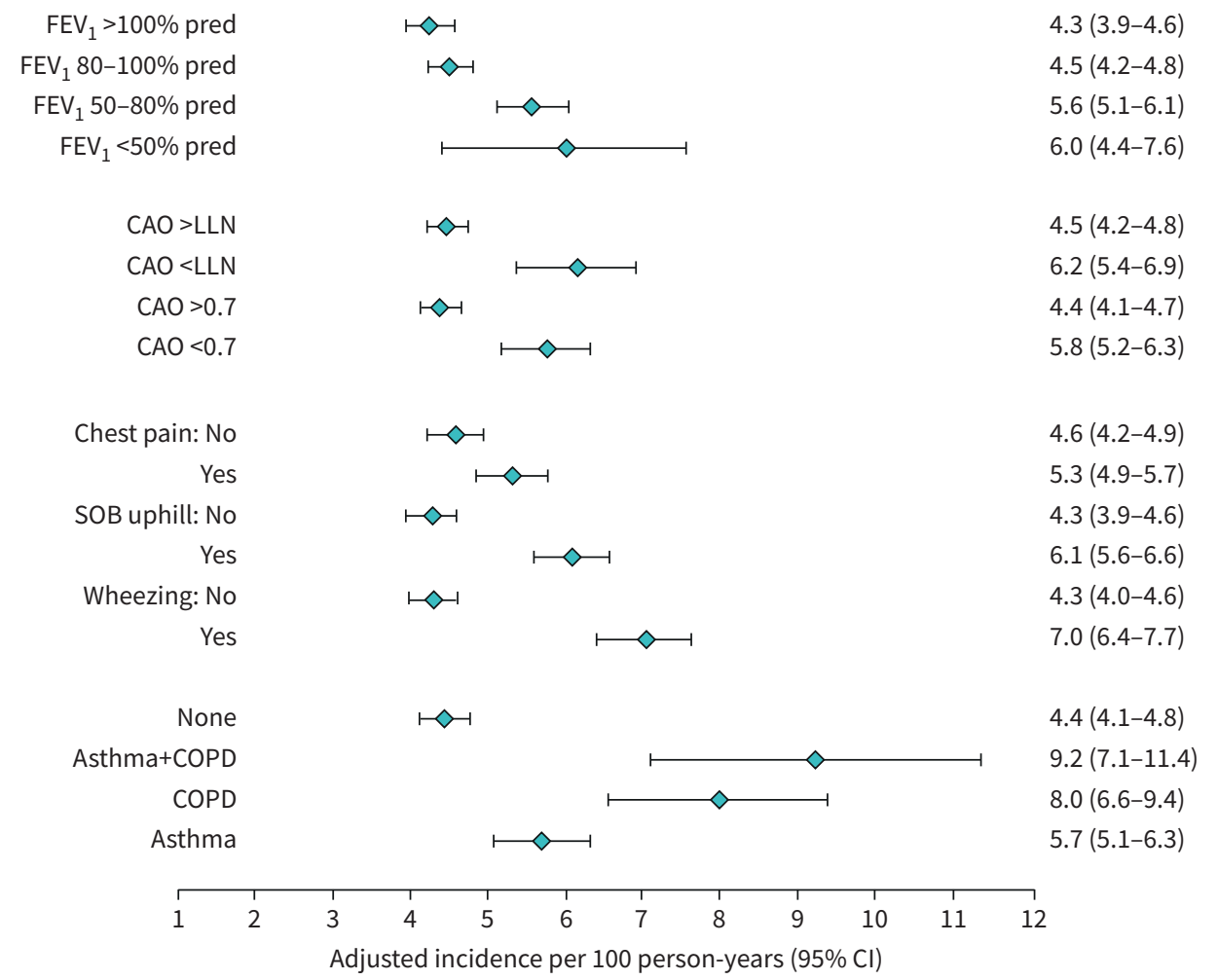

FIGURE 3 Adjusted (age, sex, smoking) incidence rates of chronic cough for respiratory symptoms, airways diseases, lung function and airflow obstruction. Data are presented as mean (95\% CI). FEV 1 : forced expiratory volume in $1 \mathrm{~s}$; CAO: chronic airflow obstruction; LLN: lower limit of normal; SOB: shortness of breath. 


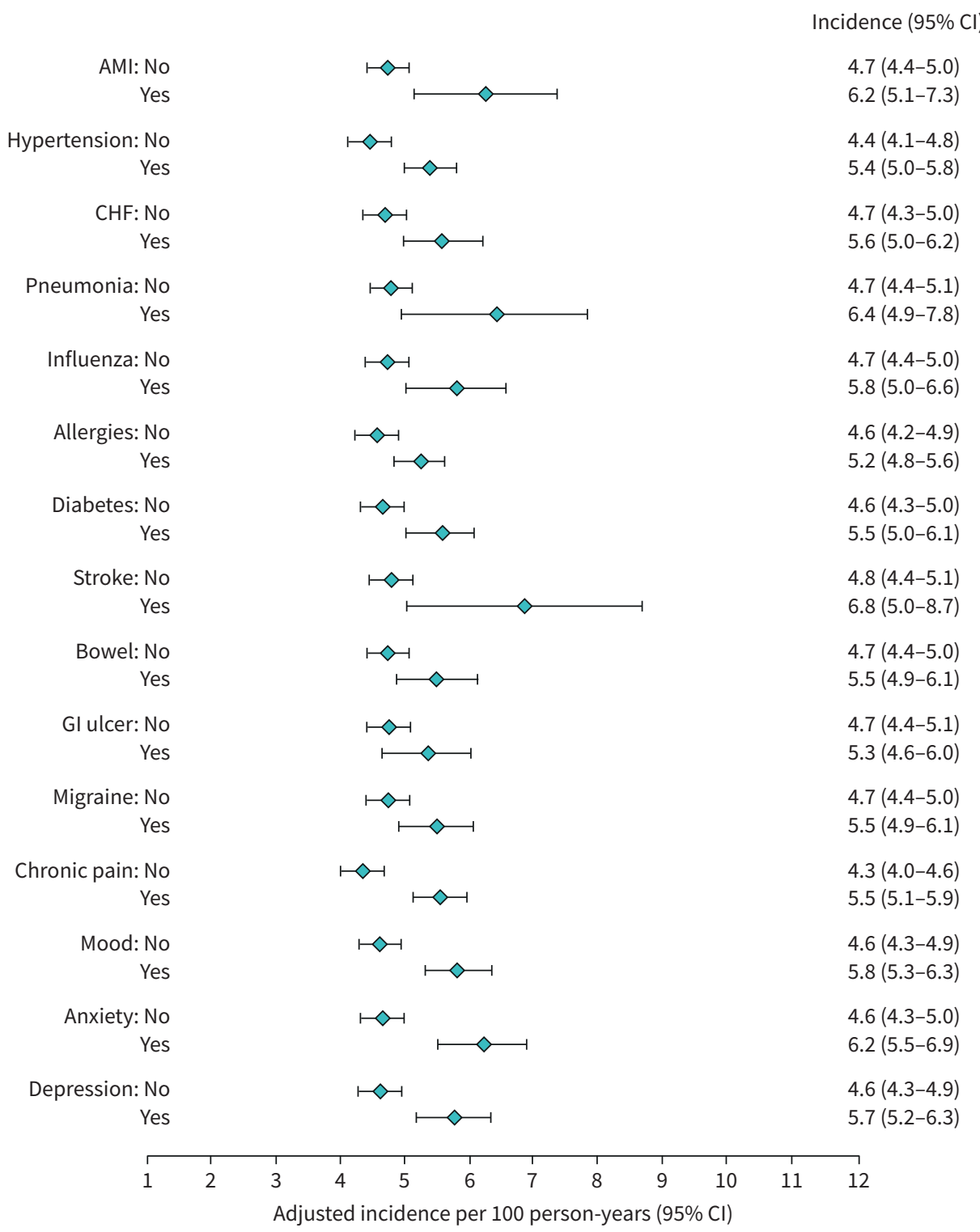

FIGURE 4 Adjusted (age, sex, smoking) incidence rates of chronic cough in the presence of other medical comorbidities. Data are presented as mean $(95 \% \mathrm{CI})$. AMI: acute myocardial infarction; CHF: congestive heart failure; GI: gastrointestinal.

smokers, and being underweight or obese. Furthermore, incidence rates were highest with wheeze, $\mathrm{FEV}_{1}$ 50-80\% pred, airflow obstruction, airway diseases (asthma/COPD) and a history of pneumonia or influenza in the past year. A history of cardiovascular diseases, mental health and mood disorders, chronic pain and diabetes were all associated with a higher incidence of chronic cough.

A prevalence of $16-18 \%$ in the CLSA sample is one of the highest globally. A meta-analysis of 90 studies showed global prevalence estimates to be $18 \%$ for Australia, $13 \%$ for Europe, $11 \%$ for the United States, $7 \%$ for Asia and 2\% for Africa [5]. The reasons for these large variations are unclear, but 19 different chronic cough definitions were identified. The most common definition was "cough $\geqslant 3$ months" or "cough $\geqslant 3$ months for two successive years" or "cough and phlegm $\geqslant 3$ months for two consecutive years". To our knowledge, six studies have used the current 8-week definition: in the UK (12\%) [15], Finland (7.2\%) [16], Copenhagen (4\%) [17], South Korea (2.6\%) [18], Japan (2.2\%) [19] and Nigeria (1.1\%) [20]. The CLSA-recruited participants were aged between 45 and 85 years at baseline, which could have influenced the high prevalence compared to the UK, Europe and the USA.

In addition, the incidence of chronic cough is much higher in the CLSA compared with the Rotterdam Study [21]. The adjusted incidence rate ranged from 3.58-5.70 per 100 person-years with increasing age, 
which is three to four times the overall incidence of 1.16 from the Rotterdam study. This was despite the fact there were more current smokers in the Rotterdam study (15.5\%) compared with the CLSA (8.5\%) and the cohort was slightly older (67.8 versus 61.5 years). A possible explanation for this difference is that the Rotterdam study had a $28.6 \%$ attrition rate (due to death or loss to follow-up) compared to $\sim 10 \%$ in the CLSA, and the former used the chronic bronchitis definition for chronic cough.

Although speciality chronic cough clinics show female predominance by $\sim 2: 1[4,13,22]$, the same pattern is not observed in population based studies. In the current study, there were more males with prevalent chronic cough $(53 \%)$, and the proportion of all males compared with females who had chronic cough was higher (15.2\% versus $12.7 \%)$. This is in contrast to the Copenhagen [17] and Rotterdam [21] studies, which demonstrated a slight female predominance. In this study, after adjusting for age, sex and smoking status, the incidence of chronic cough was still slightly higher in males, but the magnitude of the difference was small. Interestingly, this is consistent with studies from South Korea [18] and China [13, 23, 24], where there is no female predominance of chronic cough. We speculate that the reasons for the female predominance in cough clinics and in clinical trials may be due to greater cough frequency, intensity, disruption and impact on quality of life in females than males [3, 25, 26]. Furthermore, socioeconomic factors such as being in full-time employment or comorbid anxiety, depression and mood disorders may influence referral to speciality clinics.

Smoking is a well-known causative factor of chronic cough $[17,21,27]$, but the fact that former smokers and never-smokers have similar prevalence of chronic cough at each age group is reassuring and suggests that smoking cessation should be the foremost target for intervention in all clinical encounters in primary and secondary care [28-30]. This study extends knowledge of the harmful respiratory manifestations of smoking by demonstrating that airflow obstruction, COPD and a lower $\mathrm{FEV}_{1} \%$ predicted are all associated with a higher prevalence of chronic cough. This is consistent with findings from the Copenhagen General Population Study [31].

Canada is a large country with different, often extreme climates and environmental exposures. The variations in the prevalence of chronic cough throughout the different urban centres suggests the possibility that weather, changes in temperature, and air pollution could influence chronic cough. It is well known that airway nerves responsible for cough express transient receptor channels which are temperature sensitive $[32,33]$, and patients in clinical practice often complain of worsening cough due to changes in temperature [34]. This neuronal dysfunction is supported by heightened and exaggerated capsaicin-evoked cough responses in chronic cough, COPD and asthma [35-37]. These provincial differences require further exploration.

The majority of participants in the CLSA are white Caucasian, but there are nearly 1000 non-white participants, and their prevalence is almost half that of the white participants. Likewise, participants born in North America/Europe/Oceania have almost double the prevalence of chronic cough. The reasons for this are unclear. Studies investigating cough reflex sensitivity have no difference between Caucasian, Indian and Chinese healthy controls [38]. There may be genetic, socioeconomic, cultural or occupational exposures which are yet to be explored.

Understanding chronic cough at a population level can provide a different perspective and may identify other associated risk factors; for example, occupational exposure to dust/fumes [17] and chronic pain [39]. This study showed many other cardiovascular and mental health conditions that were associated with a higher incidence of chronic cough in a population. A potential common factor linking cerebrovascular accidents, hypertension, acute myocardial infarct and diabetes is that angiotensin-converting enzyme (ACE) inhibitors are used in all these conditions and is the commonest medication to cause chronic cough as a side-effect [40]. Stroke can result in swallowing difficulties with an increased risk of aspiration. Anxiety and depression are well-known associated risk factors in speciality clinics, but we have now shown this in a population-based study. In addition, there may exist a shared mechanistic pathology between chronic pain and chronic cough, where an impairment in descending inhibitory neurons has been described in both conditions $[41,42]$. The type of mood disorders increasing the risk of chronic cough is unknown, but may help in the clinical assessment and treatment of patients with chronic cough.

There are limitations to this study. First, the CLSA used a 12-month definition of chronic cough rather than 8 weeks. The 8 -week cut-off is arbitrary and although useful to initiate investigations for a cause, has no inherent diagnostic or prognostic value. Recent clinical trials of chronic cough have an inclusion criteria of chronic cough $>12$ months $[43,44]$. An amendment to the CLSA was made in 2018 to ask participants whether daily cough has lasted $>8$ weeks, $>1$ year or $>5$ years. These data will be collected in future follow-up visits and help to reduce any recall bias. Second, there are currently no questions on heartburn, indigestion, nasal congestion, runny nose or a physician diagnosis of gastro-oesophageal reflux disease or chronic rhinosinusitis. This does not allow identification of other potential associated conditions 
of chronic cough. Third, medication data such as inhaler therapy, proton-pump inhibitors, nasal steroids and ACE inhibitors are currently not available to identify those with potentially refractory chronic cough. Fourth, the CLSA sample represents the population living $25-50 \mathrm{~km}$ from the 11 data collection sites across seven provinces, and hence is not representative of the whole country. However, sites were across the provinces. Fifth, individuals visiting speciality clinics for chronic cough are likely to have more severe symptoms or are to experience more negative outcomes than those who do not attend speciality clinics $[10,13]$. Thus, population-based studies may not be generalisable to patients in chronic cough clinics.

\section{Conclusions}

The prevalence of chronic cough in Canada is high at 16-18\%, with geographic and ethnic differences. The prevalence increases with age, is more common in males and in current smokers. After adjusting for age, sex and smoking, the incidence of chronic cough is higher in males, increases with age and being underweight or obese. The presence of respiratory, cardiovascular, mental health disorders, chronic pain and diabetes were all associated with a higher incidence of chronic cough.

Acknowledgements: This research was made possible using the data/biospecimens collected by the Canadian Longitudinal Study on Aging (CLSA). Funding for the CLSA is provided by the Government of Canada through the Canadian Institutes of Health Research under grant reference LSA 94473 and the Canada Foundation for Innovation. This research has been conducted using the CLSA dataset, Baseline and Follow-up 1 Comprehensive Dataset, under Application Number 1909024. The CLSA is led by Parminder Raina, Christina Wolfson and Susan Kirkland. The opinions expressed in this manuscript are the authors' own and do not reflect the views of the CLSA. The final manuscript was reviewed and approved by the Publication Review Committee of the CLSA.

Data availability: Data are available from the Canadian Longitudinal Study on Aging (CLSA) (www.clsa-elcv.ca) for researchers who meet the criteria for access to de-identified CLSA data.

Author contributions: I. Satia, A.J. Mayhew, K.J. Killian, P.M. O’Byrne and P. Raina contributed substantially to the study concept and design. All authors made substantial contributions to the acquisition, analysis or interpretation of data for the manuscript. I. Satia, A.J. Mayhew and N. Sohel contributed substantially to drafting the manuscript. All authors contributed to the critical revision of the manuscript for important intellectual content, final approval of the version to be published, and agreement to be accountable for all aspects of the work in ensuring that questions related to the accuracy or integrity of any part of the work are appropriately investigated and resolved. P. Raina had full access to all of the data in the study, and takes responsibility for the integrity of the data and the accuracy of the data analysis.

Conflict of interest: I. Satia reports grants and personal fees from Merck during the conduct of the study; and personal fees for educational talks for general practitioners from GSK and AstraZeneca, grants and personal fees from Merck Canada, an ERS Respire 3 Marie Curie Fellowship and an E.J. Moran Campbell Early Career Award, outside the submitted work. A.J. Mayhew has nothing to disclose. N. Sohel has nothing to disclose. O. Kurmi has nothing to disclose. K.J. Killian has nothing to disclose. P.M. O’Byrne reports grants and personal fees from AstraZeneca and Medimmune, personal fees from GSK and Chiesi, and grants from Novartis, outside the submitted work. P. Raina has nothing to disclose.

Support statement: The study was funded by Merck Canada. The study sponsor had no role in the design and conduct of the study; collection, management, analysis, and interpretation of the data; preparation, review, or approval of the manuscript; and decision to submit the manuscript for publication. I. Satia is currently supported by the E.J. Moran Campbell Early Career Award, Dept of Medicine, McMaster University. Funding information for this article has been deposited with the Crossref Funder Registry.

\section{References}

1 Morice AH, Millqvist E, Bieksiene K, et al. ERS guidelines on the diagnosis and treatment of chronic cough in adults and children. Eur Respir J 2020; 55: 1901136.

2 Irwin RS, French $\mathrm{CL}$, Chang $\mathrm{AB}$, et al. Classification of cough as a symptom in adults and management algorithms: CHEST guideline and expert panel report. Chest 2018; 153: 196-209.

3 French CL, Irwin RS, Curley FJ, et al. Impact of chronic cough on quality of life. Arch Intern Med 1998; 158 $1657-1661$.

4 Chamberlain SA, Garrod R, Douiri A, et al. The impact of chronic cough: a cross-sectional European survey. Lung 2015; 193: 401-408.

5 Song WJ, Chang YS, Faruqi S, et al. The global epidemiology of chronic cough in adults: a systematic review and meta-analysis. Eur Respir J 2015; 45: 1479-1481.

6 Birring S, Prudon B, Carr A, et al. Development of a symptom specific health status measure for patients with chronic cough: Leicester Cough Questionnaire (LCQ). Thorax 2003; 58: 339-343.

7 Johansson $\mathrm{H}$, Johannessen $\mathrm{A}$, Holm $\mathrm{M}$, et al. Prevalence, progression and impact of chronic cough on employment in Northern Europe. Eur Respir J 2021; 57: 2003344.

8 Vernon M, Kline Leidy N, Nacson A, et al. Measuring cough severity: development and pilot testing of a new seven-item cough severity patient-reported outcome measure. Ther Adv Respir Dis 2010; 4: 199-208.

9 Irwin RS, Curley FJ, French CL. Chronic cough. The spectrum and frequency of causes, key components of the diagnostic evaluation, and outcome of specific therapy. Am Rev Respir Dis 1990; 141: 640-647.

10 Zeiger RS, Schatz M, Butler RK, et al. Burden of specialist-diagnosed chronic cough in adults. J Allergy Clin Immunol Pract 2020; 8: 1645-1657.

11 Raina PS, Wolfson C, Kirkland SA, et al. The Canadian longitudinal study on aging (CLSA). Can J Aging 2009; 28: $221-229$. 
12 Raina P, Wolfson C, Kirkland S, et al. Cohort profile: the Canadian Longitudinal Study on Aging (CLSA). Int J Epidemiol 2019; 48: 1752-1753j.

13 Morice AH, Jakes AD, Faruqi S, et al. A worldwide survey of chronic cough: a manifestation of enhanced somatosensory response. Eur Respir J 2014; 44: 1149-1155.

14 Canadian Longitudinal Study of Aging (CLSA). CLSA Technical Document 2020: Sampling and Computation of Response Rates and Sample Weights for the Tracking (Telephone Interview) Participants and Comprehensive Participants. 2020. www.clsa-elcv.ca/doc/3965

15 Ford AC, Forman D, Moayyedi P, et al. Cough in the community: a cross sectional survey and the relationship to gastrointestinal symptoms. Thorax 2006; 61: 975-979.

16 Lätti AM, Pekkanen J, Koskela HO. Defining the risk factors for acute, subacute and chronic cough: a cross-sectional study in a Finnish adult employee population. BMJ Open 2018; 8: e022950.

17 Çolak Y, Nordestgaard BG, Laursen LC, et al. Risk factors for chronic cough among 14,669 individuals from the general population. Chest 2017; 152: 563-573.

18 Kang MG, Song WJ, Kim HJ, et al. Point prevalence and epidemiological characteristics of chronic cough in the general adult population: the Korean National Health and Nutrition Examination Survey 2010-2012. Medicine 2017; 96: e6486.

19 Fujimura M. Frequency of persistent cough and trends in seeking medical care and treatment - results of an internet survey. Allergol Int 2012; 61: 573-581.

20 Desalu O, Salami A, Fawibe A. Prevalence of cough among adults in an urban community in Nigeria. West Afr J Med 2011; 30: 337-341.

21 Arinze JT, de Roos EW, Karimi L, et al. Prevalence and incidence of, and risk factors for chronic cough in the adult population: the Rotterdam Study. ERJ Open Res 2020; 6: 00300-2019.

22 Everett CF, Kastelik JA, Thompson RH, et al. Chronic persistent cough in the community: a questionnaire survey. Cough 2007; 3: 5.

23 Long L, Lai K. Characteristics of Chinese chronic cough patients. Pulm Pharmacol Ther 2019; 57: 101811.

24 Lai K, Long L, Yi F, et al. Age and sex distribution of Chinese chronic cough patients and their relationship with capsaicin cough sensitivity. Allergy Asthma Immunol Res 2019; 11: 871-884.

25 Dicpinigaitis P. Prevalence of urinary incontinence in women with chronic cough. Chest 2019; 155: Suppl., 300A.

26 Ajith AK, Rekha A, Duttagupta S, et al. Prevalence and factors of urinary incontinence among postmenopausal women attending the obstetrics and gynecology outpatient service in a tertiary health care center in Kochi, Kerala. Indian J Community Med 2019; 44: S30-S33.

27 Sumner H, Woodcock A, Kolsum U, et al. Predictors of objective cough frequency in chronic obstructive pulmonary disease. Am J Respir Crit Care Med 2013; 187: 943-949.

28 Farooqi MAM, Cheng V, Wahab M, et al. Investigations and management of chronic cough: a 2020 update from the European Respiratory Society Chronic Cough Task Force. Pol Arch Intern Med 2020; 130: 789-795.

29 Satia I, Badri H, Al-Sheklly B, et al. Towards understanding and managing chronic cough. Clin Med 2016; 16 s92-s97.

30 Smith JA, Woodcock A. Chronic cough. N Engl J Med 2017; 376: 183-184.

31 Landt E, Çolak Y, Lange P, et al. Chronic cough in individuals with COPD: a population-based cohort study Chest 2020; 157: 1446-1454.

32 Mazzone SB, Undem BJ. Vagal afferent innervation of the airways in health and disease. Physiol Rev 2016; 96: 975-1024.

33 Huang J, Zhang X, McNaughton PA. Modulation of temperature-sensitive TRP channels. Semin Cell Dev Biol 2006; 17: 638-645.

34 Hilton E, Marsden P, Thurston A, et al. Clinical features of the urge-to-cough in patients with chronic cough. Respir Med 2015; 109: 701-707.

35 Hilton EC, Baverel PG, Woodcock A, et al. Pharmacodynamic modeling of cough responses to capsaicin inhalation calls into question the utility of the C5 end point. J Allergy Clin Immunol 2013; 132: 847-855.

36 Satia I, Tsamandouras N, Holt K, et al. Capsaicin-evoked cough responses in asthmatic patients: evidence for airway neuronal dysfunction. J Allergy Clin Immunol 2017; 139: 771-779.

37 Belvisi MG, Birrell MA, Khalid S, et al. Neurophenotypes in airway diseases. Insights from translational cough studies. Am J Respir Crit Care Med 2016; 193: 1364-1372.

38 Dicpinigaitis PV, Allusson VR, Baldanti A, et al. Ethnic and gender differences in cough reflex sensitivity. Respiration 2001; 68: 480-482.

39 Arinze JT, Verhamme KM, Luik AI, et al. The interrelatedness of chronic cough and chronic pain. Eur Respir J 2021; 57: 2002651.

40 Dicpinigaitis PV. Angiotensin-converting enzyme inhibitor-induced cough: ACCP evidence-based clinical practice guidelines. Chest 2006; 129: 169S-173S.

41 Hilton E, Satia I, Holt K, et al. The effect of pain conditioning on experimentally evoked cough: evidence of impaired endogenous inhibitory control mechanisms in refractory chronic cough. Eur Respir J 2020; 56: 2001387.

42 Lewis GN, Rice DA, McNair PJ. Conditioned pain modulation in populations with chronic pain: a systematic review and meta-analysis. J Pain 2012; 13: 936-944.

43 Smith JA, Kitt MM, Morice AH, et al. Gefapixant, a P2X3 receptor antagonist, for the treatment of refractory or unexplained chronic cough: a randomised, double-blind, controlled, parallel-group, phase $2 \mathrm{~b}$ trial. Lancet Respir Med 2020; 8: 775-785.

44 Smith JA, Kitt MM, Butera P, et al. Gefapixant in two randomised dose-escalation studies in chronic cough. Eur Respir J 2020; 55: 1901615. 